\title{
CALCULATION OF RESPONSE SPECTRA FROM STRONG-MOTION EARTHQUAKE RECORDS
}

\author{
By Navin C. Nigam and Paul C. Jennings
}

\section{ABSTRACT}

\begin{abstract}
A numerical method for computing response spectra from strong-motion earthquake records is developed, based on the exact solution to the governing differential equation. The method gives a three to four-fold saving in computing time compared to a third order Runge-Kutta method of comparable accuracy. An analysis also is made of the errors introduced at various stages in the calculation of spectra so that allowable errors can be prescribed for the numerical integration. Using the proposed method of computing or other methods of comparable accuracy, example calculations show that the errors introduced by the numerical procedures are much less than the errors inherent in the digitization of the acceleration record.
\end{abstract}

\section{INTRODUCTION}

Since their introduction (Benioff, 1934; Biot, 1941; Housner, 1941), response spectra of strong-motion earthquakes have proved useful and informative in problems of design and analysis of structures subjected to strong earthquake motions. The spectra, calculated from the recorded ground acceleration, are plots of the maximum response to the earthquake of a simple oscillator over a range of values of its natural period and damping. These curves provide a description of the frequency characteristics of the ground motion and give the maximum response of simple structures to the earthquake. By superposition of different modes of response, spectrum techniques can be applied to the design and analysis of complex structures such as buildings and dams. Used in this manner the spectrum technique represents an approach intermediate between a design based on static loads and a complete integration of the equations of motion of the complex structure.

Strong-motion earthquake records have been obtained infrequently in the past and the reduction to digital form, or equivalent analog form, and subsequent calculation of spectra have been performed on more or less an individual basis. However, in recent years the number of strong-motion instruments in the seismic regions of the world, particularly California, Mexico and Japan, has increased to the point where a major earthquake in these areas will generate a large number of records. The potential volume of the data and the development of the tape-recording accelerograph indicate clearly that rapid and automated data processing and spectrum calculation procedures are needed. In an effort to fulfill part of this need, this paper presents a rapid, accurate method for computation of response spectra from strong-motion earthquake records.

Response spectra were first obtained by Biot (1941), using a direct mechanical analog and later by Housner and McCann (1949) using electric analog techniques. The availability of digital computers and a progressive increase in the speed of digital computation in recent years has led to an increasing use of digital computers in the calculation of spectra. The extensive application of the response spectrum to problems in earthquake engineering has sustained interest in methods of calculating spectra and has raised questions regarding accuracy, reproducibility and economy in such calculations (Hudson, 1962; Brady, 1966; Berg, 1963; Schiff and Bogdanoff, 1967).

The digital computation of spectra requires the repeated numerical solution of the 
response of a simple oscillator to a component of recorded ground acceleration. The motion of the oscillator is described by a second order, linear, inhomogeneous differential equation, and if a digital description of the earthquake record is available, the response can be obtained by numerical integration. Several numerical integration techniques have been used for calculation of spectra, for example, the third order RungeKutta scheme of integration has been preferred by some investigators because of its accuracy, long-range stability, self-starting feature and because it can be adapted easily for use when the excitation is not defined at regular intervals (Jennings, 1963; Hildebrand, 1956). The truncation error in this method is proportional to $(\Delta \theta)^{4}$, where $\Delta \theta$ is the normalized interval of integration. Thus by a suitable choice of $\Delta \theta$ the calculations can be performed to an acceptable degree of accuracy.

An alternative approach to calculation of spectra is based on obtaining the exact solution to the governing differential equation for the successive linear segments of the excitation, then using this solution to compute the response at discrete time intervals in a purely arithmetical way (Hudson, 1962; Iwan, 1960). This method does not introduce numerical approximations in the integration other than those inherent in roundoff, so in this sense it is an exact method.

In this paper this method is used to develop a computation technique which leads to a three-to four-fold saving in computing time compared to a third order RungeKutta method of comparable accuracy. If the earthquake record is digitized at equal time intervals, the proposed scheme gives spectral values which are exact in the sense mentioned above. If the record is digitized at arbitrary time intervals, however, it is necessary to introduce an approximation into the digitization. An analysis of the errors introduced at various stages in the preparation of response spectra and the results of digitization experiments show that the additional numerical approximations are not detrimental.

The numerical techniques for calculating response spectra have been codified into computer programs in Fortran IV; listings of these programs and details not included herein are available in a recent report (Nigam and Jennings, 1968).

\section{Formulation of the Method}

Spectra are defined by the maximum response of a simple oscillator subjected to base acceleration $a(t)$ as shown in Figure 1. The equation of motion of the oscillator is

$$
\ddot{x}+2 \beta \omega \dot{x}+\omega^{2} x=-a(t)
$$

in which $\beta=$ the fraction of critical damping and $\omega=$ the natural frequency of vibrations of the oscillator.

Assuming that $a(t)$ may be approximated by a segmentally linear function as shown in Figure 2, equation (1) may be written as

$$
\ddot{x}+2 \beta \omega \dot{x}+\omega^{2} x=-a_{i}-\frac{\Delta a_{i}}{\Delta t_{i}}\left(t-t_{i}\right) ; \quad t_{i} \leqq t \leqq t_{i+1}
$$

with

$$
\begin{gathered}
\Delta t_{i}=t_{i+1}-t_{i} \\
\Delta a_{i}=a_{i+1}-a .
\end{gathered}
$$


The solution of equation (2), for $t_{i} \leqq t \leqq t_{i+1}$ is given by

$$
\begin{gathered}
x=e^{-\beta \omega\left(t-t_{i}\right)}\left[C_{1} \sin \omega \sqrt{1-\beta^{2}}\left(t-t_{i}\right)+C_{2} \cos \omega \sqrt{1-\beta^{2}}\left(t-t_{i}\right)\right] \\
-\frac{a_{i}}{\omega^{2}}+\frac{2 \beta}{\omega^{3}} \frac{\Delta a_{i}}{\Delta t_{i}}-\frac{1}{\omega^{2}} \frac{\Delta a_{i}}{\Delta t_{i}}\left(t-t_{i}\right)
\end{gathered}
$$

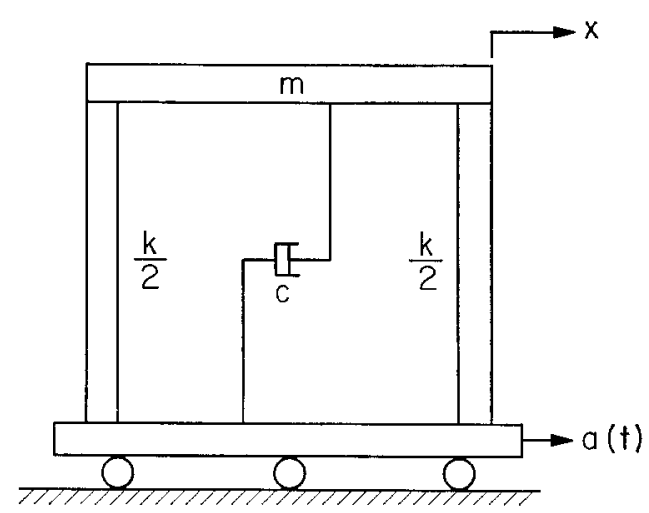

Frg. 1. A simple oscillator.

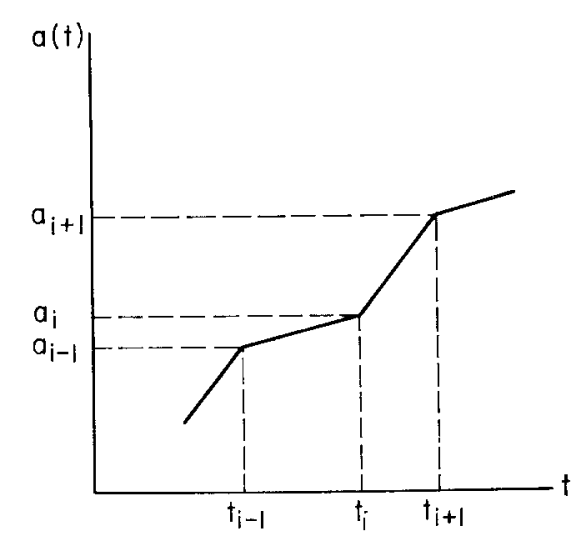

FIG. 2. Idealized base acceleration.

in which $C_{1}$ and $C_{2}$ are constants of integration. Setting $x=x_{i}$ and $\dot{x}=\dot{x}_{i}$ at $t=t_{i}$ and solving for $C_{1}$ and $C_{2}$, it is found that

$$
\begin{aligned}
& C_{1}=\frac{1}{\omega \sqrt{1-\beta^{2}}}\left(\beta \omega x_{i}+\dot{x}_{i}-\frac{2 \beta^{2}-1}{\omega^{2}} \frac{\Delta a_{i}}{\Delta t_{i}}+\frac{\beta}{\omega} a_{i}\right) \\
& C_{2}=x_{i}-\frac{2 \beta}{\omega^{3}} \frac{\Delta a_{i}}{\Delta t_{i}}+\frac{a_{i}}{\omega^{2}} .
\end{aligned}
$$

Substitution of these values of $C_{1}$ and $C_{2}$ into equation (4) will show that $x$ and $\dot{x}$ at $t=t_{i+1}$ are given by

$$
\bar{x}_{i+1}=A\left(\beta, \omega, \Delta t_{i}\right) \bar{x}_{i}+B\left(\beta, \omega, \Delta t_{i}\right) \bar{a}_{i}
$$


in which

$$
\begin{aligned}
& \bar{x}_{i}=\left\{\begin{array}{l}
x_{i} \\
\dot{x}_{i}
\end{array}\right\} \quad \bar{a}_{i}=\left\{\begin{array}{l}
a_{i} \\
a_{i+1}
\end{array}\right\} \\
& A=\left[\begin{array}{ll}
a_{11} & a_{12} \\
a_{21} & a_{22}
\end{array}\right] \quad B=\left[\begin{array}{ll}
b_{11} & b_{12} \\
b_{21} & b_{22}
\end{array}\right] .
\end{aligned}
$$

The elements of matrices $A$ and $B$ are given by

$$
\begin{aligned}
& a_{11}=e^{-\beta \omega \Delta t_{i}}\left(\frac{\beta}{\sqrt{1-\beta^{2}}} \sin \omega \sqrt{1-\beta^{2}} \Delta t_{i}+\cos \omega \sqrt{1-\beta^{2}} \Delta t_{i}\right) \\
& a_{12}=\frac{e^{-\beta \omega \Delta t_{i}}}{\omega \sqrt{1-\beta^{2}}} \sin \omega \sqrt{1-\beta^{2}} \Delta t_{i} \\
& a_{21}=-\frac{\omega}{\sqrt{1-\beta^{2}}} e^{-\beta \omega \Delta t_{i}} \sin \omega \sqrt{1-\beta^{2}} \Delta t_{i} \\
& a_{22}=e^{-\beta \omega \Delta t_{i}}\left(\cos \omega \sqrt{1-\beta^{2}} \Delta t_{i}-\frac{\beta}{\sqrt{1-\beta^{2}}} \sin \omega \sqrt{1-\beta^{2}} \Delta t_{i}\right) \\
& b_{11}=e^{-\beta \omega \Delta t_{i}}\left[\left(\frac{2 \beta^{2}-1}{\omega^{2} \Delta t_{i}}+\frac{\beta}{\omega}\right) \frac{\sin \omega \sqrt{1-\beta^{2}} \Delta t_{i}}{\omega \sqrt{1-\beta^{2}}}\right. \\
& \left.+\left(\frac{2 \beta}{\omega^{3} \Delta t_{i}}+\frac{1}{\omega^{2}}\right) \cos \omega \sqrt{1-\beta^{2}} \Delta t_{i}\right]-\frac{2 \beta}{\omega^{3} \Delta t_{i}} \\
& b_{12}=-e^{-\beta \omega \Delta t_{i}}\left[\left(\frac{2 \beta^{2}-1}{\omega^{2} \Delta t_{i}}\right) \frac{\sin \omega \sqrt{1-\beta^{2}} \Delta t_{i}}{\omega \sqrt{1-\beta^{2}}}+\frac{2 \beta}{\omega^{3} \Delta t_{i}} \cos \omega \sqrt{1-\beta^{2}} \Delta t_{i}\right] \\
& -\frac{1}{\omega^{2}}+\frac{2 \beta}{\omega^{3} \Delta t_{i}} \\
& b_{21}=e^{-\beta \omega \Delta i_{i}}\left[\left(\frac{2 \beta^{2}-1}{\omega^{2} \Delta t_{i}}+\frac{\beta}{\omega}\right)\left(\cos \omega \sqrt{1-\beta^{2}} \Delta t_{i}-\frac{\beta}{\sqrt{1-\beta^{2}}} \sin \omega \sqrt{1-\beta^{2}} \Delta t_{i}\right)\right. \\
& -\left(\frac{2 \beta}{\omega^{3} \Delta t_{i}}+\frac{1}{\omega^{2}}\right)\left(\omega \sqrt{1-\beta^{2}} \sin \omega \sqrt{1-\beta^{2}} \Delta t_{i}+\beta \omega \cos \omega \sqrt{1-\beta^{2}} \Delta t_{i}\right) \\
& +\frac{1}{\omega^{2} \Delta t_{i}} \\
& b_{22}=-e^{-\beta \omega \Delta t_{i}}\left[\frac{2 \beta^{2}-1}{\omega^{2} \Delta t_{i}}\left(\cos \omega \sqrt{1-\beta^{2}} \Delta t_{i}-\frac{\beta}{\sqrt{1-\beta^{2}}} \sin \omega \sqrt{1-\beta^{2}} \Delta t_{i}\right)\right. \\
& \left.-\frac{2 \beta}{\omega^{3} \Delta t_{i}}\left(\omega \sqrt{1-\beta^{2}} \sin \omega \sqrt{1-\beta^{2}} \Delta t_{i}+\beta \omega \cos \omega \sqrt{1-\beta^{2}} \Delta t_{i}\right)\right] \\
& -\frac{1}{\omega^{2} \Delta t_{i}}
\end{aligned}
$$


From equation (2), it follows that the absolute acceleration, $\ddot{z}_{i}$, of the mass at time $t_{i}$ is given by

$$
\ddot{z}_{i}=\ddot{x}_{i}+a_{i}=-\left(2 \beta \omega \dot{x}_{i}+\omega^{2} x_{i}\right) .
$$

Hence, if the displacement and velocity of the oscillator are known at some time $t_{0}$, the state of the oscillator at all subsequent times $t_{i}$ can be computed exactly by a step-by-step application of equations (6) and (7). The computational advantage of this approach lies in the fact that $A$ and $B$ depend only on $\beta$, $\omega$ and $\Delta t_{i}$. $\beta$ and $\omega$ are constant during the calculation of each spectrum value, and if $\Delta t_{i}$ is constant also, $x_{i}, \dot{x}_{i}$ and $\ddot{z}_{i}$ can be evaluated by the execution of only ten multiplication operations for each step of integration. Matrices $A$ and $B$, defined by the rather complicated expressions equations $(6 \mathrm{~d})$ and (6e) need to be evaluated only at the beginning of each spectrum calculation.

If varying time intervals are used, it is necessary, in general, to compute $A$ and $B$ at each step of integration. However, by rounding the time coordinates of the record, as discussed below, the number of these matrices needed during the calculation can be reduced to only a few. These, too can be computed at the beginning of the calculation and called upon when needed, thereby saving computing time.

\section{Computation of Spectra}

To construct the response spectra, it is necessary to find the maximum values of the displacement, velocity and acceleration during a given excitation. This is done by computing the response at discrete time intervals through equations (6) and (7) and monitoring the response parameters to retain the maximum values. Thus, the response spectra are given by

$$
\begin{aligned}
& S_{d}(\omega, \beta)=\operatorname{Max}_{i=1, N}\left[x_{i}(\omega, \beta)\right] \\
& S_{v}(\omega, \beta)=\operatorname{Max}_{i=1, N}\left[\dot{x}_{i}(\omega, \beta)\right] \\
& S_{a}(\omega, \beta)=\operatorname{Max}_{i=1, N}\left[\ddot{z}_{i}(\omega, \beta)\right]
\end{aligned}
$$

in which $S_{d}, S_{v}$ and $S_{a}$ are the spectral values of displacement, velocity, and acceleration respectively, for selected values of damping and natural frequency; and $N$ is the total number of discrete points at which the response is obtained.

This process of obtaining the maximum response is approximate because the response is found only at discrete points, whereas the true maxima may occur between such points. This error, called the error of discretization, is inherent in all numerical procedures, but can be bounded within acceptable limits by a suitable choice of the time interval. The discretization error operates to give spectrum values lower than the true values and the error will be a maximum if the maximum response occurs exactly midway between two discrete points as shown in Figure 3. An estimate for the upper bound of this error can be found by noting that at the time of maximum displacement or velocity, the response of the oscillator is nearly sinusoidal at a frequency near its natural frequency. Under this assumption the error can be related to the maximum interval of integration $(\Delta \tau)_{m}$ and the period of the oscillator as illustrated in Figure 3. 
Digitization of Earthquake Records. When earthquake records are digitized at equal time intervals, the acceleration values are measured at regular time steps and are assumed to be connected by straight line segments. If the interval of digitization, $\Delta t$, is sufficiently small, this procedure approximates the actual earthquake record quite closely. The choice of the interval of digitization depends upon the period range of interest and the nature of the earthquake record; commonly used values range from 0.01 to 0.04 seconds. Analytical methods for assessing the error induced by such a sampling process are available (Schiff and Bogdanoff, 1967, Bendat and Piersol, 1966). Digitization at equal time intervals will be a virtual necessity for efficient processing of accelerograms recorded on magnetic tape, and in other instances where analog to digital conversion is made automatically.

For records digitized at unequal time intervals the abscissae and ordinates of the points where changes of slope are judged to occur are measured and the points are again assumed to be connected by straight line segments (Figure 2). This procedure leads to variable time intervals between successive points. The accuracy of this ap-

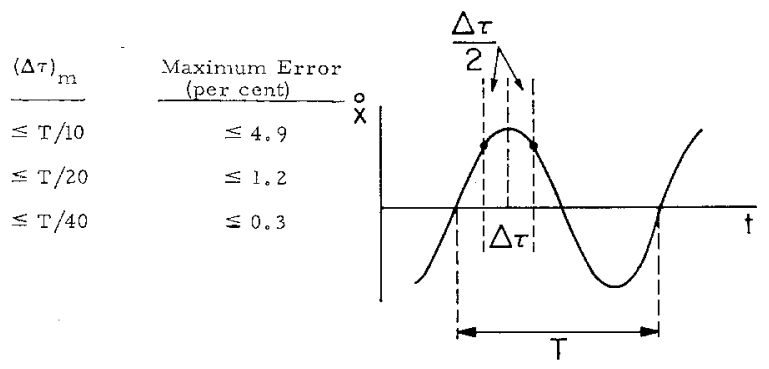

Fig. 3. Error due to discretization.

proach is difficult to determine analytically, but should be considerably greater than would be achieved by the same number of sample points at equal intervals. This method is the most convenient for manually controlled digitization techniques.

Spectra Computation for Equal Time Intervals. Equations (6) show that matrices $A$ and $B$ are functions of $\omega, \beta$ and $\Delta t_{i}$. If $\Delta t_{i}$ is constant, that is, the earthquake record is digitized at equal time intervals, these matrices need to be computed only once for each pair of $\omega$ and $\beta$ and the chosen value of $\Delta \tau$, the interval of integration. Also, since the method does not involve truncation error, it is possible to use larger intervals of integration than in other methods. The choice of the interval of integration, $\Delta \tau$, is controlled by the interval of digitization $(\Delta \tau \leqq \Delta t)$ and the error of discretization. Figure 3 shows that the error due to discretization is less than 1.2 per cent if $\Delta \tau \leqq T / 20(T=$ Natural Period). Considering other errors in the computation of spectra, this choice of interval of integration seems reasonable. In fact, $\Delta \tau \leqq T / 10$ may be satisfactory for most purposes.

Since the third order Runge-Kutta scheme of integration often has been used for the digital computation of spectra, the proposed method is compared to it with regard to accuracy and computing time. To make the comparison, undamped velocity spectrum values were obtained for artificial earthquake No. 7 (Jennings, 1963) using a third order Runge-Kutta scheme, for four values of the interval of integration: $\Delta \tau \leqq T / 10$, $T / 20, T / 40$, and $T / 80$. The spectrum values also were obtained by the present method for $\Delta \tau \leqq T / 10$ and $T / 20$. The results of these computations are compiled in Table 1 , which shows the spectral values and the relative values of the computation time for different cases. 
The data in Table 1 show that it is necessary to use $\Delta \tau \leqq T / 80$ to get accuracy to three significant figures using the third order Runge-Kutta scheme of integration. For the exact scheme of integration, however, $\Delta \tau \leqq T / 10$ may be acceptable for most practical purposes. It is seen also from the last two columns of Table 1 that in some cases the spectral values are the same for $\Delta \tau \leqq T / 10$ and $\Delta \tau \leqq T / 20$. This occurs when the maximum value has a time coordinate which is a common multiple of both step lengths.

The relative times given in Table 1 indicate that for accuracy to three significant figures the proposed method with $\Delta \tau \leqq T / 20$ is about 3 to 4 times as fast as the third

TABLE 1

Undamped Vetocity Spectra and Computing Times for Records Digitized at Equal Time Intervals

(Artificial Earthquake No. 7, $\Delta t=0.025$ secs)

\begin{tabular}{|c|c|c|c|c|c|c|}
\hline \multirow{3}{*}{ Period In Secs } & \multicolumn{6}{|c|}{$S_{v}$ In Ins/Sec } \\
\hline & \multicolumn{4}{|c|}{ third order Runge-Kuica } & \multicolumn{2}{|c|}{ exact } \\
\hline & $\Delta \tau \leqq T / 10$ & $\Delta \tau \leqq T / 20$ & $\Delta \tau \leqq T / 40$ & $\Delta \tau \leqq T / 80$ & $\Delta \boldsymbol{\tau} \leqq T / 10$ & $\Delta \tau \leqq T / 20$ \\
\hline 0.05 & 0.126427 & 0.151835 & 0.167647 & 0.204386 & 0.213610 & 0.213611 \\
\hline 0.1 & 1.175386 & 1.700338 & 1.938206 & 2.000499 & 2.021378 & 2.021378 \\
\hline \multirow[t]{2}{*}{0.2} & 5.892718 & 6.974802 & 7.951382 & 8.077665 & 8.129632 & 8.142673 \\
\hline & $\cdots$ & & & & $\cdots$ & \\
\hline 0.3 & 8.149913 & 8.262451 & 7.951037 & 7.951037 & 7.927181 & 7.9925 .54 \\
\hline \multirow[t]{2}{*}{0.4} & 16.399755 & 21.987390 & 22.985190 & 23.097921 & 23.174661 & 23.174610 \\
\hline & & $\cdots \cdots \cdots$ & & & & \\
\hline 0.5 & 20.716002 & 20.716002 & 22.897381 & 23.164824 & 23.352803 & 23.352803 \\
\hline 0.7 & 25.625738 & 25.625738 & 26.732634 & 26.861891 & 26.966020 & 26.966020 \\
\hline \multirow[t]{2}{*}{0.9} & 14.911507 & 14.911507 & 15.019002 & 15.024426 & 15.059319 & 15.059319 \\
\hline & & & $\cdots \cdots \cdots$ & & & \\
\hline 1.0 & 13.712175 & 13.712175 & 13.712175 & 13.809191 & 13.841665 & 13.841665 \\
\hline 1.6 & 23.740771 & 23.740771 & 23.740771 & 23.757607 & 23.772265 & 23.772265 \\
\hline 2.0 & 13.137178 & 13.137178 & 13.137178 & 13.137178 & 13.148899 & 13.148899 \\
\hline $\begin{array}{l}\text { Relative com- } \\
\text { putation } \\
\text { time }\end{array}$ & 1.5 & 2 & 3 & 4 & .75 & 1 \\
\hline
\end{tabular}

Note: Spectral values below dotted lines were obtained at $\Delta \tau=0.025$ secs, the interval of digitization.

order Runge-Kutta technique. Using the IBM 7094 Computer at the California Institute of Technology Computing Center, the computing time for the proposed method with $\Delta \tau \leqq T / 20$ averages about 1.0 sec execution time per spectrum point for the 30 sec record used to compile Table 1.

Spectra Computation for Unequal Time Intervals. If the earthquake record is digitized at unequal time intervals, matrices $A\left(\omega, \beta, \Delta t_{i}\right)$ and $B\left(\omega, \beta, \Delta t_{i}\right)$ in equations (6) will, in general, change from step to step, making it necessary to compute them anew at each step of integration. From equations $(6 \mathrm{~d})$ and $(6 \mathrm{e})$, which define the elements of these matrices, it is clear that this computation would take a large amount of time; in fact, it would make the method slower than a comparable third order Runge-Kutta scheme. However, the inherent limitations of the digitized earthquake record make it possible to develop an approximate method of satisfactory accuracy which gives a saving in computing time. The essential feature of this method is a rounding of the time coordinates of the original record to a predetermined accuracy. Then, choosing an 
appropriate maximum interval of integration, $(\Delta \tau)_{m}$, and subdividing each time interval of the rounded time record into time intervals equal to or less than $(\Delta \tau)_{m}$, the calculation proceeds much as before, except that $A\left(\omega, \beta, \Delta \tau_{j}\right)$ and $B\left(\omega, \beta, \Delta \tau_{j}\right)$ must be calculated for four or five different values of $\Delta \tau_{j}$. This procedure is illustrated in Table 2.

After the time record is rounded and subdivided, the values of the ground acceleration at the additional points created by subdivision are computed by linear interpolation from the original record. The modified record now consists of a set of points along the time axis, spaced at a limited number of known intervals, and the corresponding values of the acceleration. The operations necessary to produce the modified record can easily be programmed for computer execution.

The number and size of the time intervals in the modified record depend on the way

TABLE 2

Round-off and Subdivision of Time Record $\left[(\Delta \tau)_{m}=0.04 \mathrm{secs}\right]$

\begin{tabular}{|c|c|c|c|c|c|c|}
\hline \multicolumn{2}{|c|}{ Step } & \multirow{2}{*}{$\begin{array}{c}\text { Original Time } \\
10.4267\end{array}$} & \multirow{2}{*}{$\frac{\text { Rounded Time }}{10.43}$} & \multicolumn{2}{|c|}{$\begin{array}{l}\text { Subdivision into Intervals } \\
\text { of Integration } \\
(\Delta \tau)_{\mathrm{m}}=0.04\end{array}$} & Remarks \\
\hline \multirow{3}{*}{ I } & $t_{i}$ & & & \multicolumn{2}{|c|}{0.04} & Round-off to .01 \\
\hline & $t_{i+1}$ & 10.5213 & 10.52 & \multicolumn{2}{|c|}{0.04} & \multirow{9}{*}{ Round-off to .005} \\
\hline & $\Delta t_{i}$ & 0.0946 & 0.09 & \multicolumn{2}{|c|}{0.01} & \\
\hline \multirow{7}{*}{ II } & & & & \multicolumn{2}{|c|}{0.09} & \\
\hline & & & & (a) & (b) & \\
\hline & $t_{i}$ & 10.4267 & 10.425 & 0.04 & 0.04 & \\
\hline & $t_{i+1}$ & 10.5213 & 10.520 & 0.04 & 0.04 & \\
\hline & \multirow[t]{3}{*}{$\Delta t_{i}$} & 0.0946 & 0.095 & 0.01 & 0.015 & \\
\hline & & & & 0.005 & 0.095 & \\
\hline & & & & 0.095 & 0.095 & \\
\hline
\end{tabular}

in which rounding of the time coordinates is carried out and the choice of $(\Delta \tau)_{m}$. For the example presented in Table 2, the possibilities are:

I $\Delta \tau_{j}=0.04,0.03,0.02$ and 0.01 (4 time-intervals)

IIa $\Delta \tau_{j}=0.04,0.03,0.02,0.01$ and 0.005 (5 time-intervals)

IIb $\Delta \tau_{j}=0.04,0.035,0.03,0.025,0.02,0.015,0.01,0.005$ (8 time-intervals).

Therefore, if the original record is modified as indicated above, the exact method needs only a limited number of matrices for each pair of $\omega$ and $\beta$, and these can be computed before the integration is started. By indexing each possible $\Delta \tau_{j}$ and the corresponding matrices, the appropriate matrices can be called at each step of integration.

The procedure outlined above requires that the time coordinates of the record be rounded to a predetermined decimal fraction. Assuming that the original digital description of the earthquake record is exact, this is an approximation which must lead to random errors in the computation of spectra. However, a digital description of an earthquake at unequal time intervals is obtained by manual, or manually controlled, reading of a graphical record and is subject to certain unavoidable errors. If the round-off is carried out in such a way that effects of the approximation so introduced 
are well within the errors inherent in the process of digitization, the process outlined above will be acceptable.

Digitization Experiment. Berg (1963) and Brady (1966) have studied the errors introduced during the process of digitization and their effect on the computation of spectra. In the absence of gross personal errors or bias, the errors arise primarily from resolution of the scaling device and from the thickness of the trace which makes the choice of points at which a change of slope occurs somewhat arbitrary. To examine these errors an experiment on digitization was conducted by three persons $(X, Y, Z)$ on the Benson-Lehner Data Reducer 099, at the California Institute of Technology. The first 5 seconds on the Taft, California N21E record were digitized independently by $X$ (5 times), $Y$ (2 times) and $Z$ (2 times), at the highest resolution of the Data Reducer for the record used $(0.0015$ secs and $0.0001 \mathrm{~g})$. In each case points were taken when a change in slope was judged to occur in the record. Taking one record of each person as a standard, corresponding time coordinates on the other records were subtracted from it to obtain what is called here the self-error of digitization. The mean and

TABLE 3

Self-Error of Digitization in Ttme Coordinates

\begin{tabular}{|c|c|c|c|c|c|c|c|}
\hline \multirow{2}{*}{$\begin{array}{l}\text { Error in Time } \\
\text { Coordinates }\end{array}$} & \multicolumn{5}{|c|}{$X$} & \multirow{2}{*}{$Y$} & \multirow{2}{*}{$Z$} \\
\hline & 1 & 2 & 3 & 4 & average & & \\
\hline Mean (sec) & -0.00101 & 0.0001 & -0.00021 & 0.00061 & -0.00041 & 0.00013 & 0.00045 \\
\hline $\begin{array}{l}\text { Standard } \\
\text { Devia- } \\
\text { tion (sec) }\end{array}$ & 0.00432 & 0.00503 & 0.00412 & 0.00426 & 0.00443 & 0.00398 & 0.00556 \\
\hline
\end{tabular}

standard deviation for each case are shown in Table 3 . The standard deviations shown in Table 3 indicate that a careful reader will be consistent within 0.004 sec about 70 per cent of the time, with a mean error very close to zero.

To compare the digitized records obtained by different persons, one of the records of $X$ was taken as an overall standard and the records of $Y$ and $Z$ were subtracted from it to obtain what is called the cross-error of digitization. The mean and standard deviation of the error are shown in Table 4. The standard deviations from the mean shown in Tables 3 and 4 indicate that the cross error is about four times the self error, thus showing the effect of personal preferences in reading. The consistent, negative means indicate a constant shift in the time axis of the record chosen as a standard as compared to the other records. Such a shift does not effect the deviation about the mean.

A comparable measure of the error introduced by rounding-off the time record can be obtained by subtracting the rounded record from the original record and computing the mean and standard deviation. These were done for one of the records digitized by $X$, and the results are shown in Table 5, for rounding to 0.01 and 0.005 sec.

The results of the experiment described in Table 5 show that the error introduced by rounding-off the time coordinates of an earthquake record to 0.01 or 0.005 secs is well within the error inherent in the process of digitization. For round-off to 0.01 secs, the standard deviation of the error due to round-off is about half of the standard deviation of the self-error of digitization and about one sixth of the standard deviation of the cross-error. For round-off to 0.005 secs, this margin is further increased by a factor of 2 . 
From this it is concluded that a round-off to 0.01 or 0.005 secs is an acceptable approximation.

Effect of Rounding Upon Accuracy. Berg (1963) has examined errors in spectra caused by random errors in time and acceleration coordinates and has concluded from an approximate analysis and from computer experimentation that a scatter of the order of 20 per cent in undamped spectra is to be expected from identical computing procedures applied to independently prepared digitizations of the same accelerogram. He attributed the scatter to random errors in reading the accelerogram. Using Berg's approximate formulae for expected error in velocity spectra due to random errors in

TABLE 4

\begin{tabular}{|c|c|c|c|c|c|c|}
\hline \multirow{2}{*}{$\begin{array}{l}\text { Error in Time } \\
\text { Coordinates }\end{array}$} & \multicolumn{3}{|c|}{$Y$} & \multicolumn{3}{|c|}{$Z$} \\
\hline & 1 & 2 & average & 1 & 2 & average \\
\hline Mean (sec) & -0.02525 & -0.02538 & -0.02532 & -0.02212 & -0.02257 & -0.02235 \\
\hline $\begin{array}{l}\text { Standard De- } \\
\text { viation (sec) }\end{array}$ & 0.01819 & 0.01794 & 0.01806 & 0.01520 & 0.01520 & 0.01520 \\
\hline
\end{tabular}

TABLE 5

\begin{tabular}{lcc}
\multicolumn{2}{c}{ Error Dut To Round-off IN Time Coordinates } \\
\hline Error in Time Coordinates & Roound-off to 0.01 secs & Round-off to 0.005 secs \\
\hline Mean (sec) & 0.00003 & 0.00004 \\
Standard Deviation (sec) & 0.00285 & 0.00149 \\
Maximum Error & \pm 0.005 & \pm 0.0025 \\
\hline
\end{tabular}

the time coordinates of the record, and assuming $a=0.045 g,\left(S_{v}\right)$ ave $\approx 20 \mathrm{sin} / \mathrm{sec}$ and $\Delta t=0.05$ secs, the following results are found:

Expected Percentage

Error in $S_{v}(D=$

Expected Error in $S_{v}$

30 secs)

$0.12 \sqrt{ } D$

3.3

$0.06 \sqrt{ } D$

1.6

Round-off to 0.005 secs

$(\sigma \approx 0.0015$ secs $)$

Here, $a=$ the r.m.s. value of the ground acceleration, $\sigma=$ standard deviation of the expected error in the time coordinates and $D=$ the duration of the earthquake. The results indicate that the errors in spectral values due to round-off to 0.01 or 0.005 secs are much less than the 17 per cent error Berg (1963) found likely to occur as a result of random errors in reading the time coordinates during digitization.

The validity of the above analysis can be checked by comparing the spectra for unrounded and rounded time records. This was done for the $\mathrm{N} 65^{\circ} \mathrm{E}$ component of the station 2 record of the Parkfield earthquake of June 27, 1966 and the N $8^{\circ} \mathbf{E}$ component of the Lima, Peru earthquake of October 17, 1966. The undamped velocity spectra for the original (unrounded) record and for the records obtained after round-off the time coordinates to 0.01 secs and to 0.005 secs are shown in Figures 4 and 5. For the Park- 
field earthquake, the three curves are almost indistinguishable. For the Lima, Peru earthquake the average percentage errors are of the order expected on the basis of Berg's approximate analysis.

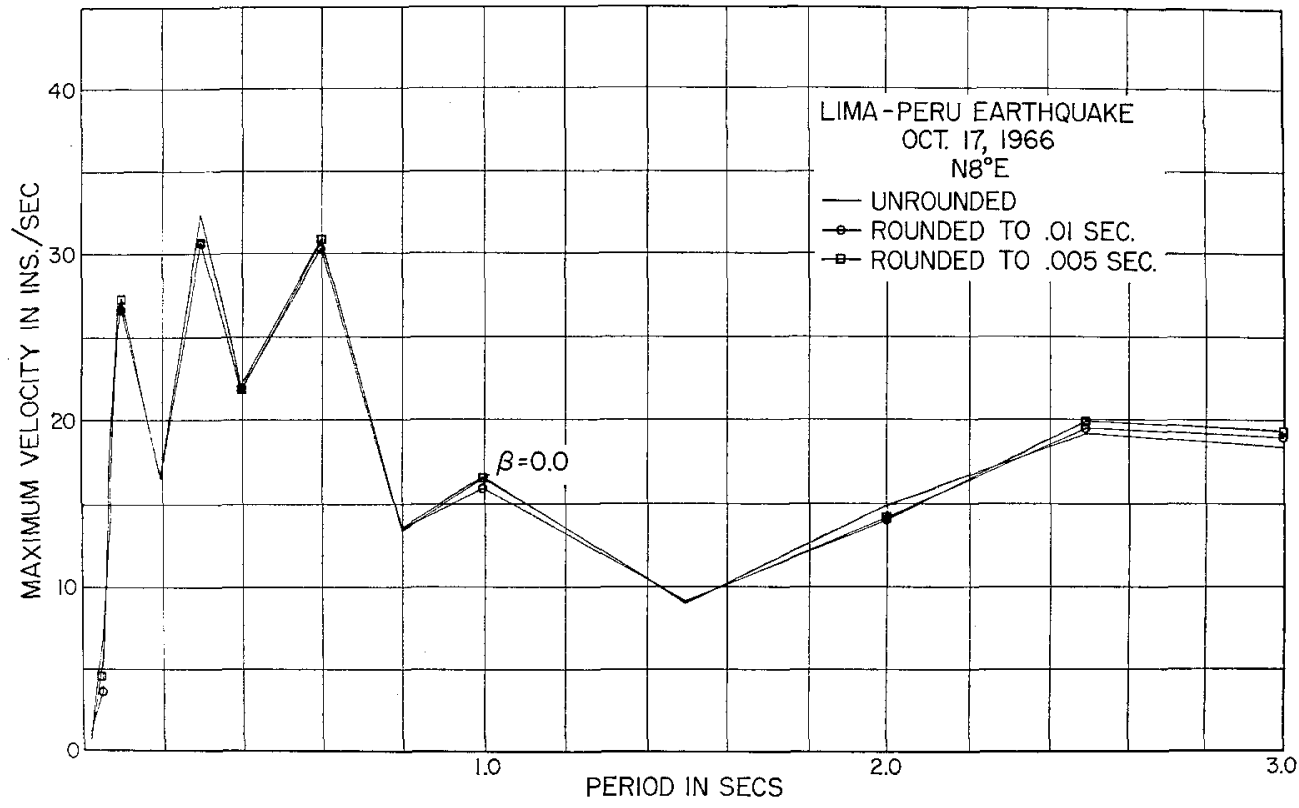

FIG. 4. Effect of rounding the time coordinates on velocity spectra.

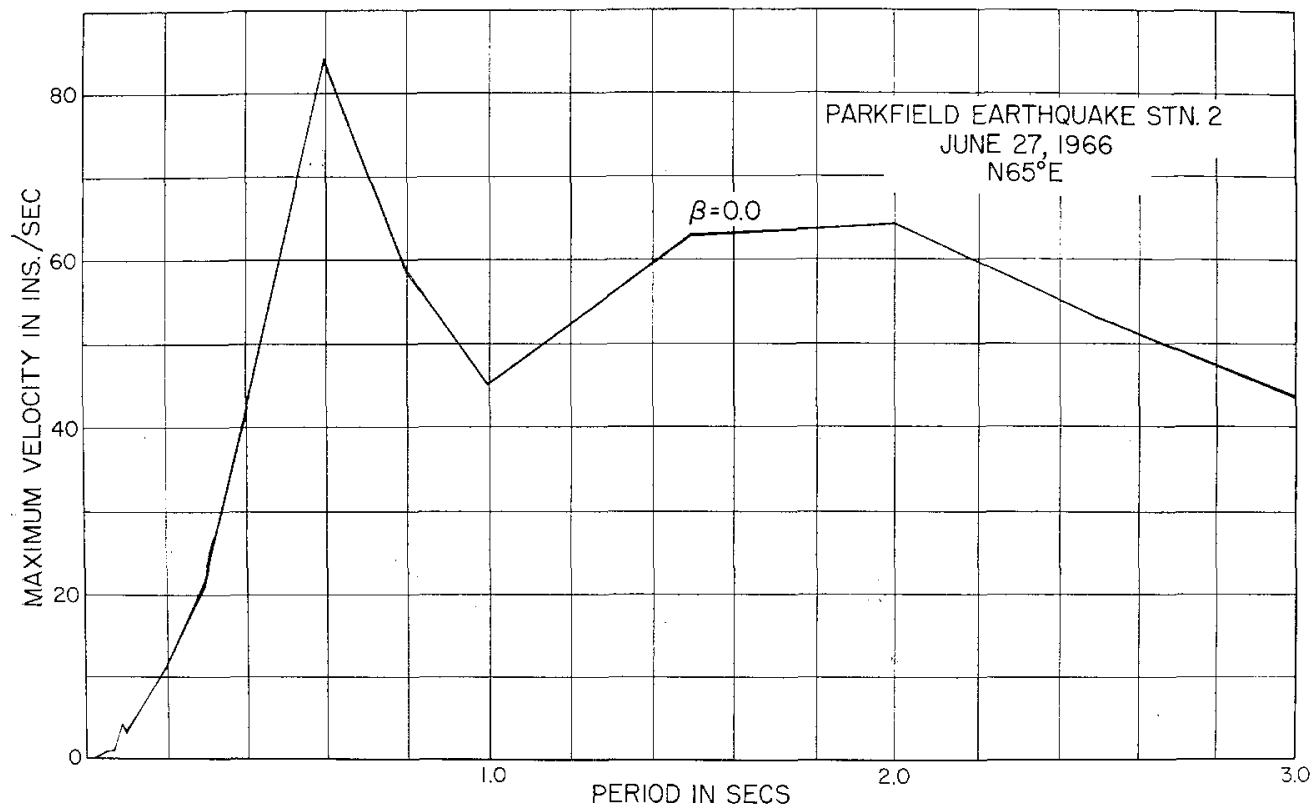

FrG. 5. Effect of rounding the time coordinates on velocity spectra.

The computing time for spectra based on digitized accelerograms rounded to 0.005 secs was found to be only 10 to 15 per cent more than the computing time for records rounded to 0.01 secs. Considering the expected error in the spectral values in the two 
cases, it is concluded that to be consistent with the choice of $(\Delta \tau)_{m}(\leqq T / 20)$, roundoff to 0.005 secs would be adopted as standard. Here again it may be pointed out that the use of $(\Delta \tau)_{m} \leqq T / 10$ and round-off to 0.01 secs should prove acceptable for most purposes.

Intervals of Integration. The procedure for computing spectra outlined above requires the choice of a maximum interval of integration, $(\Delta \tau)_{m}$. Since truncation error is not involved, the choice is governed by the error introduced by discretization, and by computing time. As shown in Figure 3 , if $(\Delta \tau)_{m} \leqq T / 20$, the error due to

TABLE 6

Undamped Velocity Spectra and Relative Computing Times for Records Digitized at Unequal Time Intervals

(N $65^{\circ} \mathrm{E}$ Component the Station 2 Accelerogram of the Parkfield Earthquake, June 27, 1966, $\left.(\Delta r)_{m}=0.05 \mathrm{secs}\right)$

\begin{tabular}{|c|c|c|c|c|c|c|}
\hline \multirow{3}{*}{ Period In Secs } & \multicolumn{6}{|c|}{$S_{v}$ In Ins/Sec } \\
\hline & \multicolumn{4}{|c|}{ Third Order Runge-Kutta } & \multicolumn{2}{|c|}{ exact } \\
\hline & $\Delta \tau \leqq T / 10$ & $\Delta \tau \leqq T / 20$ & $\tau \Delta \leqq T / 40$ & $\Delta \tau \leqq T / 80$ & $\Delta \tau \leqq T / 10$ & $\Delta \tau \leqq T / 20$ \\
\hline 0.05 & 0.404177 & 0.547537 & 0.690496 & 0.781693 & 0.776551 & 0.802090 \\
\hline 0.1 & 1.864953 & 2.749153 & 3.177131 & 3.391490 & 3.446407 & 3.446375 \\
\hline 0.2 & 8.662501 & 10.587279 & 10.905353 & 10.929761 & 11.007311 & 11.007311 \\
\hline 0.3 & 14.519660 & 20.197662 & 20.780575 & 20.880682 & 20.724359 & 20.954274 \\
\hline 0.4 & 39.198212 & 40.554802 & 40.660100 & 40.677461 & 40.844893 & 40.844893 \\
\hline 0.5 & 46.064539 & 50.050687 & 51.059478 & 51.129117 & 51.329105 & 51.329105 \\
\hline 0.7 & 85.185941 & 86.373600 & 89.262830 & 89.655182 & 90.064254 & 90.063806 \\
\hline 0.9 & 57.705933 & 59.043586 & 60.146103 & 60.228546 & 60.508116 & 60.488801 \\
\hline 1.0 & 44.625701 & 44.625701 & 44.980583 & 44.990469 & 45.149522 & 45.149522 \\
\hline 1.6 & 63.948129 & 63.948129 & 63.960555 & 63.941084 & 64.027008 & 64.027008 \\
\hline 2.0 & 64.084503 & 64.084503 & 64.084503 & 64.134411 & 64.167577 & 64.167577 \\
\hline $\begin{array}{l}\text { Relative com- } \\
\text { putation } \\
\text { time }\end{array}$ & 1.25 & 1.5 & 2.25 & 3.5 & 0.75 & 1 \\
\hline
\end{tabular}

Note: Spectrum values below dotted lines were calculated for $(\Delta \tau)_{m}=0.05$ secs.

discretization is less than 1.2 per cent. This may be treated as one constraint on the choice of $(\Delta \tau)_{m}$.

The effect of $(\Delta \tau)_{m}$ on the computing time is more complex in that a larger value of $(\Delta \tau)_{m}$ tends to reduce the number of integration steps but increases the number of matrices to be calculated. Smaller values of $(\Delta \tau)_{m}$ imply the reverse results, so a choice of $(\Delta \tau)_{m}$ should strike a balance between the two effects. The ideal choice would depend on the nature of the accelerogram used, but a compromise satisfactory for most uses was achieved by the choice of $(\Delta \tau)_{m}=0.05$ for spectrum periods equal or exceeding one second and $(\Delta \tau)_{m} \leqq T / 20$ for periods less than one second.

If the time-coordinates of the record are rounded to 0.005 secs, there is more than one way in which the intervals of digitization can be subdivided, as illustrated in Table 2. The choice of subdividing method is governed by the computing time, and to examine this question a few trials were conducted using both the methods in Table 2 . It was found that in most cases, subdivision method IIa $\left(\Delta \tau_{j}=0.04,0.03,0.02,0.01\right.$ and 0.005 ) required less computing time. 
Comparison to a Third Order Runge-Kutta Method. To indicate the effect of truncation error on spectrum values obtained by a Runge-Kutta method and to compare the accuracy and computing time for that method with the techniques developed above, undamped velocity spectra were computed by both methods. The results of these computations are compiled in Table 6 .

The results in Table 6 show again that the accuracy of the proposed method is only achieved by the Runge-Kutta method with $\Delta \tau \leqq T / 80$, and that there is a three-tofour-fold savings in time by the use of the proposed technique.

Computer Programs. Computer programs, in Fortran IV, for the computation of spectra using the method of computation presented in this paper are included in a recent report (Nigam and Jennings, 1968). Flow charts and instructions for the use of the programs are given also.

\section{Conclusions}

This paper presents a numerical method for the calculation of response spectra from strong-motion earthquake accelerograms. The method is based on the exact solution of the differential equation governing the response of a simple oscillator to segmentally linear excitation. To adapt the exact solution to efficient computing, the solution is written in the form of two $2 \times 2$ matrices which operate upon the conditions at the beginning of the integration step and upon the acceleration at the beginning and end of the integration step to produce the exact velocity and displacement at the end of the time interval. Because the matrices are functions only of the damping and period of the oscillator used to calculate spectra and of the magnitude of the time interval, only a limited number of the matrices need be evaluated. The method can be applied directly to accelerograms digitized at equal time intervals, and by suitably rounding the time coordinates, to records digitized at unequal time intervals. The errors introduced by rounding the time coordinates are much less than other errors inherent in the digitization of earthquake records.

The choice of using equal or unequal time intervals for digitization of accelerograms usually is not based on computational ease, but on the type of equipment available for the work. However, the choice of digitization method affects the calculation technique and the computing time. For the same number of sample points, digitization at unequal time intervals represents the original record better than does digitization at equal intervals, and if digitization is done at equal intervals a small time step usually must be used to avoid significant distortion of the record. Because for the usual integration methods the interval of integration must be less than or equal to the interval of digitization, a small digitization interval makes it necessary to use a small integration step where otherwise a larger interval could be used. This leads to an increase in computing time and is of particular significance to the method presented herein, because this technique does not involve truncation error and therefore can be used accurately with time intervals as large as $T / 10$ to $T / 20$. Thus, an accelerogram digitized at unequal intervals, with its smaller number of coordinates, can be processed more rapidly by the proposed method than can the same record digitized at equal time intervals.

For the accelerograms used as examples in this study, the proposed method showed a three-to four-fold saving in time over a third order Runge-Kutta method of comparable accuracy. This was found to be the case for records digitized at both equal and unequal time intervals. For accelerograms with durations of 30 seconds, spectra for 30 periods and 4 values of damping were calculated and plotted by an IBM 7094 computing system with an average computing time of 120 seconds, of which 100 seconds were used for execution of the calculations. If spectra for several earthquakes were to 
be computed in the same operation, a further savings in computing time could be achieved by storing the matrices used in the integration method rather than recomputing them at the beginning of the processing of each accelerogram.

Because of the increasing interest in the response of yielding structures to earthquake motions, it is appropriate to point out that the method presented herein can be adapted also to the calculation of spectra for bilinear hysteretic and elasto-plastic oscillators. To apply the method it would be necessary to compute two sets of matrices corresponding to the two stiffness coefficients of the structure.

\section{ACKNOWLEDGMenT}

Appreciation is extended to the National Seience Foundation for partial support of this research under NSF Grant 1197X.

\section{REFERENCES}

Alford, J. L., G. W. Housner, G. W. and R. R. Martel (1951). Spectrum Analysis of Strong-Motion Earthquakes, Earthquake Engr. Res. Lab., Calif. Inst. of Tech., Pasadena. (Revised, Aug. 1964)

Bendat and A. G. Piersol (1966). Measurement and Analysis of Random Data. John Wiley and Sons, New York.

Benioff, H. (1934). Calculation of the response of an oscillator to arbitrary ground motion, Bull. Seism. Soc. of Am. 31, 398-403.

Berg, G. V. (1963). A study of errors in response spectrum analyses, Jornadas Chilenas de Sesmologia e Ingenieria Antisismica 1, B1.3, 1-11.

Berg, G. V., G. W. Housner (1961). Integrated velocity and displacement of strong earthquake ground motion, Bull. Seism. Soc. of Am. 5l, 175-189.

Biot, M. A. (1941). A mechanical analyzer for the prediction of earthquake stresses, Bull. Seism. Soc. of Am. 31, 151-171.

Brady, A. G. (1966), Studies of Response to Earthquake Ground Motion, Earthquake Engr. Res. Lab., Calif. Inst. of Tech., Pasadena.

Hildebrand, F. B. (1956). Introduction to Numerical Analysis, MeGraw-Hill Book Company Inc., New York.

Housner, G. W. (1941). Calculation of the response of an oscillator to arbitrary ground motion, Bull. Seism. Soc. of Am. 31, 143-149.

Housner, G. W. and G. D. McCann (1949). The analysis of strong-motion earthquake records with electric analog computer, Bull. Seism. Soc. of Am.39, 47-56.

Hudson, D. E. (1962). Some problems in the application of spectrum techniques to strong-motion earthquake analysis, Bull. Seism. Soc. of Am. 52, 417-430.

Iwan, W. D. (1960). Digital Calculation of Response Spectra and Fourier Spectra, Unpublished Note, Calif. Inst. of Tech., Pasadena.

Jennings, P. C. (1963). Velocity Spectra of the Mexican Earthquakes of 11 May and 19 May 1962, Earthquake Engr. Res. Lab. Calif. Inst. of Tech., Pasadena.

Nigam, N. C. and P. C. Jennings (1968). Digital Calculation of Response Spectra from StrongMotion Earthquake Records, Earthquake Engr. Res. Lab., Calif. Inst. of Tech., Pasadena.

Schiff, A. and J. L. Bogdanoff (1967). Analysis of current methods of interpreting strong motion accelograms, Bull. Seism. Soc. of Am. 57, 857-874.

Division of Engineering and Applied Science

California Institute of Technology

Pasadena, California 91109

Manuscript received September 12, 1968. 